\title{
AN ELECTROCARDIOGRAPHIC STUDY OF THE HEART IN LOBAR PNEUMONIA ${ }^{1}$
}

\author{
By ARTHUR C. DeGRAFF, JANET G. TRAVELl, and J. Allen yAGER \\ (From the Departments of Medicine of New York and Cornell Universities, and from \\ the Third (New York University) Medical Division and the Second (Cornell \\ University) Medical Division of Bellevue Hospital, New York)
}

(Received for publication June 9, 1931)

\section{INTRODUCTION}

Numerous electrocardiographic studies have been made in rheumatic fever, diphtheria and other acute infectious diseases $(1,2,3,4,5)$, but a search of the literature yields relatively little information on the changes in the heart depicted by the electrocardiogram in lobar pneumonia. Cohn and Jamieson (6) in a report on the action of digitalis in this disease discussed the tracings obtained from fifty patients not treated with digitalis. Although certain facts can be ascertained from a study of such a group, it is nevertheless true that a much larger series gives a more comprehensive picture. Furthermore, Cohn and Jamieson selected largely the mild cases to serve as controls, while those who were severely ill received digitalis. Therefore, it seemed advisable to study a large unselected group of patients to whom no digitalis had been given.

The data forming the basis of this report were obtained during the course of an investigation on the action of digitalis in lobar pneumonia. The method of selection of cases, the routine established, and the precautions observed are given in detail by Wyckoff, DuBois and Woodruff (7). Suffice it to say, that an absolutely unprejudiced random sample was obtained for both digitalis treated and untreated groups. Electrocardiograms were taken daily during the acute or febrile period of the disease, and every other day during convalescence.

${ }^{1}$ Read before the New York University Medical Society, October 4, 1930, and the Section of Medicine, New York Academy of Medicine, December 16, 1930. 
A control tracing was taken on all treated cases before any digitalis was administered. During the three years of the study 7,480 electrocardiograms were obtained on 975 patients, of whom 562 received no digitalis. On 101 patients (10.4 per cent) only one tracing, and on 89 (9.1 per cent) two tracings were taken. For the remaining 785 (80.5 per cent), the average number of electrocardiograms per patient was nine. During the first year a Cambridge portable machine was used, and in the second and third years, a Hindle Mobile No. 3.

Since this study deals with the electrocardiographic findings, uninfluenced by treatment (notably digitalis), occurring in lobar pneumonia, the analyses which follow are based on the tracings obtained from 562 patients who received no digitalis and on the control tracings from 413 digitalis treated cases, taken before any digitalis had been administered. The study of the abnormal basic rhythms however, is based, as will be explained later, on all electrocardiograms taken on the $\mathbf{9 7 5}$ digitalis treated and untreated cases.

The material to be presented lends itself to division into three main groups. They are: (1) heart rate, (2) abnormalities of rhythm and (3) abnormalities of conduction. The abnormalities of rhythm will be further subdivided into (a) the abnormal basic rhythms and (b) interruptions of the normal basic rhythm.

\section{Heart rate}

Clinicians have for years attached great importance to the heart rate in lobar pneumonia. A very rapid rate is usually associated with a severe infection and is generally regarded as indicative of a poor prognosis. Bullowa (8) has used this experience in establishing a severity rating in lobar penumonia. He attached relative numerical values to degrees' of cyanosis, respiratory rate, bacteremia, etc., as well as to the heart rate, in an attempt to forecast the prognosis. The increased severity of the disease with the higher heart rates was acknowledged by a deduction of five points from the patient's rating for every increase of ten heart beats above 110 per minute.

No one would question that the electrocardiogram is the most reliable source for the determination of the ventricular rate. Our data therefore, afford an excellent opportunity for the study of the heart rate in lobar pneumonia. Occasionally, the first tracing shows 
a slightly higher rate than subsequent ones, probably due to the apprehension of the patient. Later, the patient is not alarmed and it is possible to record a somewhat lower heart rate which varies between rather narrow extremes.

It can be seen from the curves of individual patients in Figures 1,

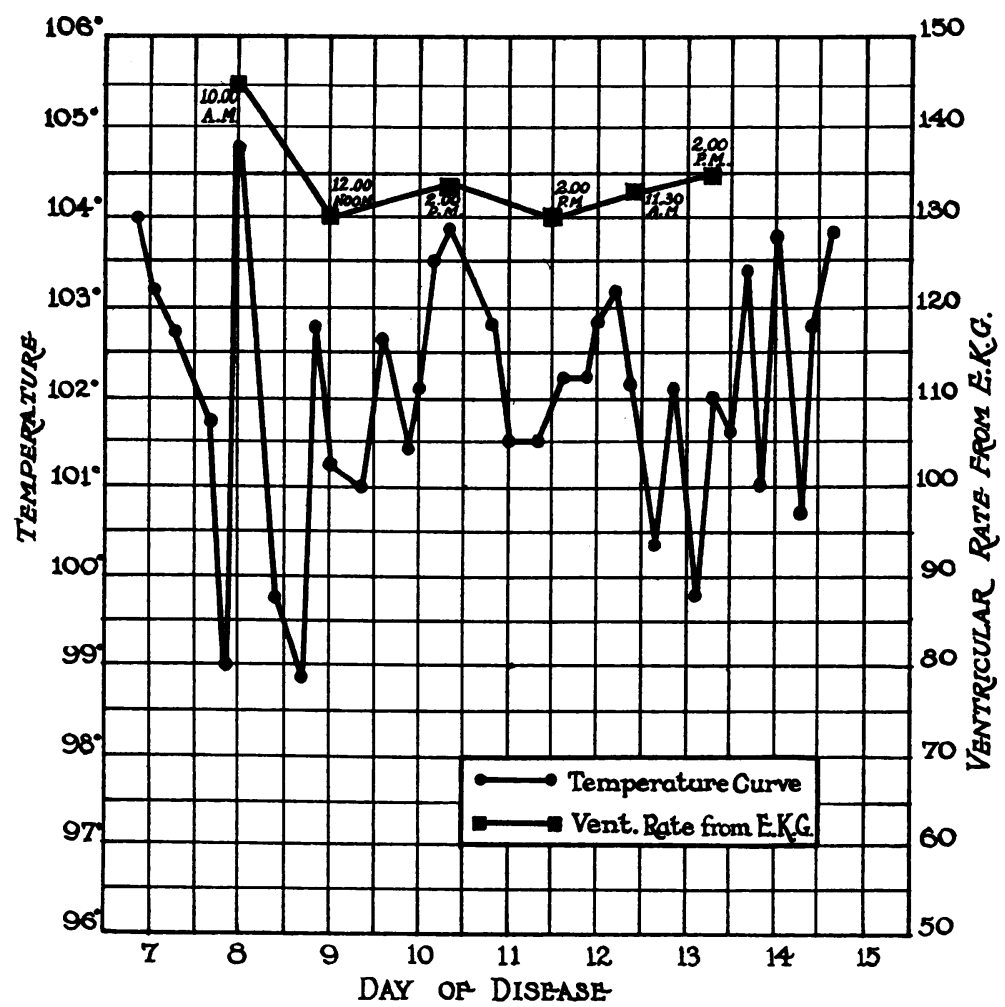

Fig. 1. Male, Age 36.

Illustrates (1) a rapid heart rate during fever (2) after the first tracing, the remarkable constancy of the heart rate from day to day in spite of fluctuations of temperature.

Note-In all figures the day of disease, as represented on the abscissae is calculated from the history of the onset.

2 and 3 that the heart rate varies within very narrow limits and that it remains within these narrow limits from day to day during the febrile period even in the presence of fluctuations of temperature. 
That such variations of heart rate are slight in a large proportion of cases is shown in Figure 4, in which the maximum range of heart rate during the febrile period for cases with sinus rhythm is plotted against the respective incidence of each. It is striking that 27 per cent showed a variation in rate of less than ten beats per minute, and that 66 per cent had a maximum range of less than twenty-one per minute.

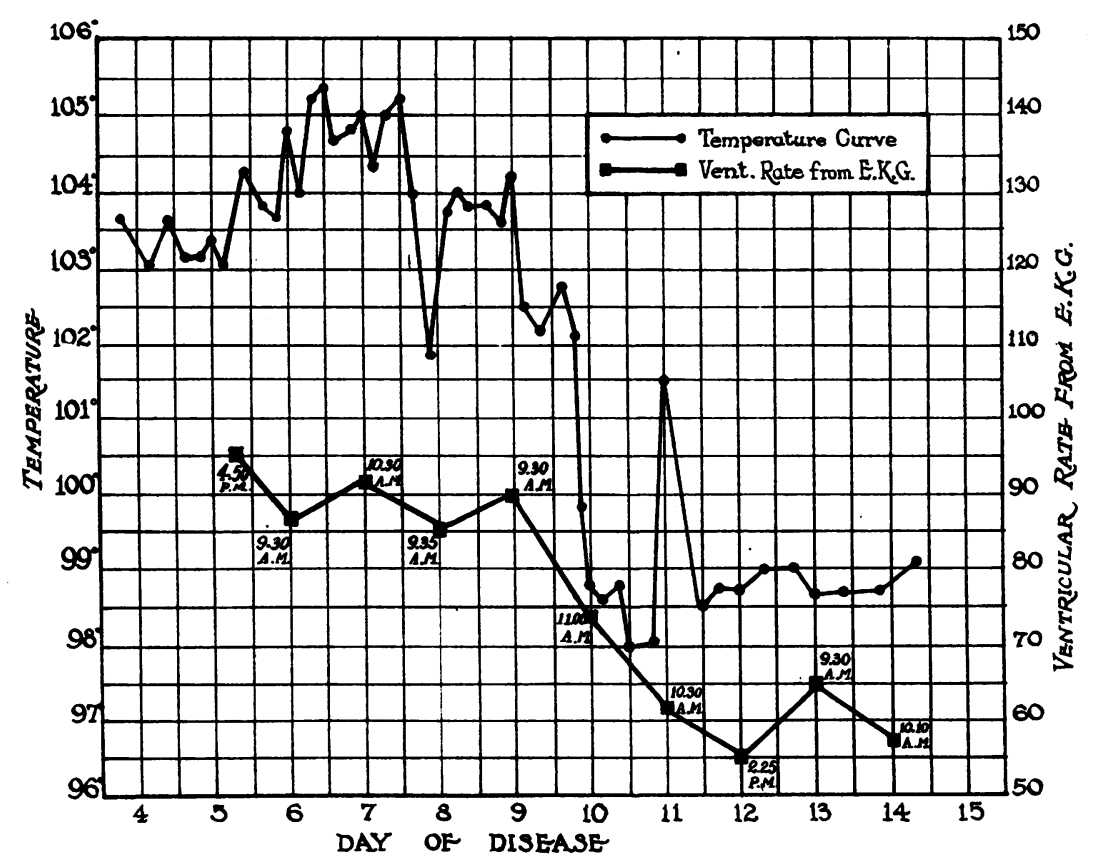

Fig. 2. Male, Age 40.

Illustrates (1) a relatively slow heart rate during fever, (2) the constancy of the heart rate during fever as calculated from the electrocardiogram, (3) sinus bradycardia after the crisis.

Where a large variation was present, it was found to be associated with one of the following conditions: (1) a premortal rise in heart rate, (2) a fall in temperature by lysis (since the range in heart rate includes the entire febrile period), and (3) a fast heart rate in the first tracing, as noted above.

It may seem possible that the truth is not reached by observations made only once in a twenty-four hour period, that variations in heart 
rate during the period between electrocardiographic observations may be missed and that the constancy in heart rate results only from the conspiracy of chance observations. Since the great majority of cases showed a relative constancy of the heart rate, this supposition is not justified. Furthermore, in six cases, whose average heart rates varied from 84 to 144 beats per minute, electrocardiograms were taken every hour over a period of eight hours. During this period the heart rates

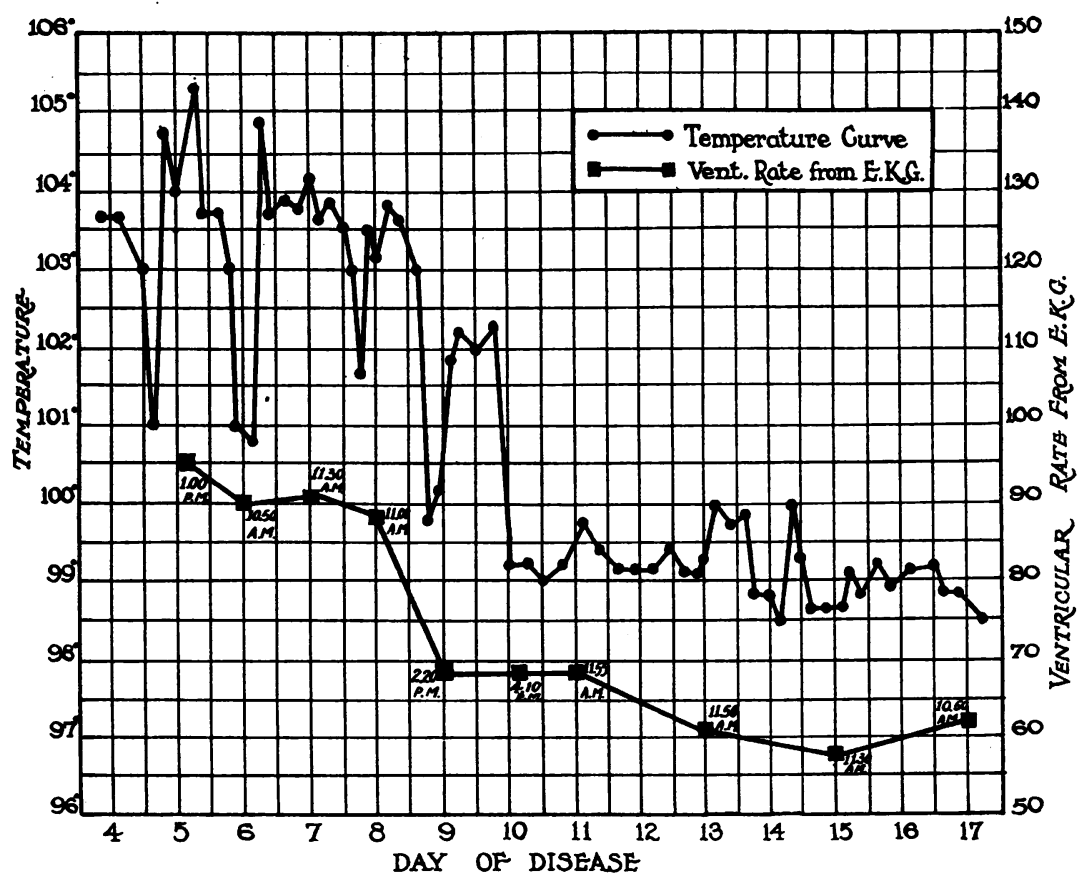

Fig. 3. Female, Age 56.

Illustrates (1) a relatively slow heart rate during fever, (2) the constancy of the heart rate during fever as calculated from the electrocardiogram.

varied from 6 to 10 beats per minute. In view of these facts, we feel satisfied that single daily observations of the heart rate (even in the presence of fluctuations of temperature) reveal the true variations. Our data furnish evidence, then, that in lobar pneumonia the variation in rate from day to day is slight.

In view of this fact. the value of heart rate as one of the important 
factors in the determination of prognosis becomes greatly increased, especially early in the disease when other clinical signs may not be clearly defined. We have, therefore, studied the ventricular rate in

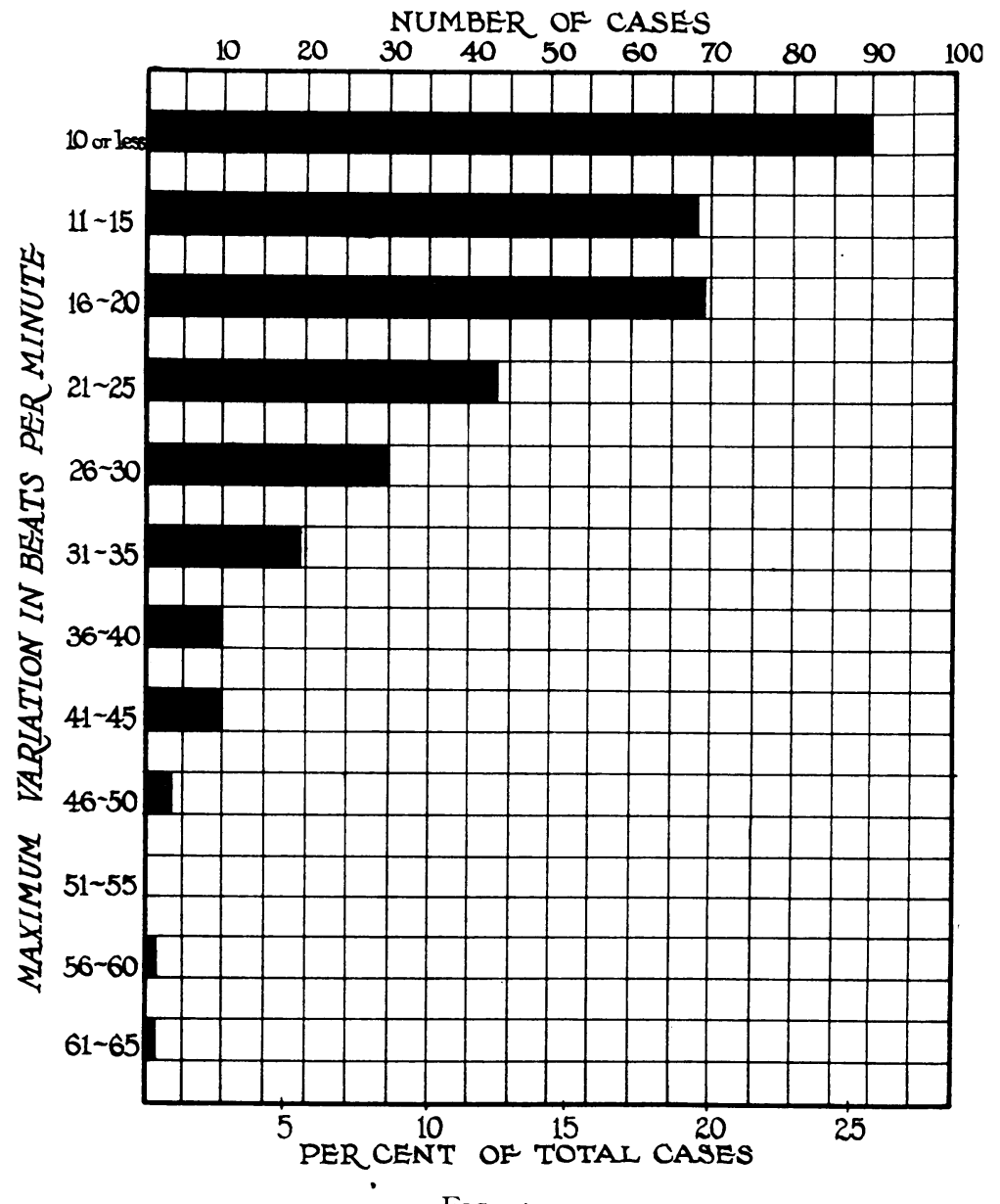

FIG. 4.

Maximum RaNcie in Heart Rate durisg fever for 349 Cases With Sinus Rhythi.

all tracings taken before crisis, lysis, or death, including the control electrocardiograms of patients treated with digitalis. A tabulation of these tracings was made according to the day of the disease on 
ARThuR C. DEgRafF, JANET G. TRAVEll, AND J. Allen Yager 639

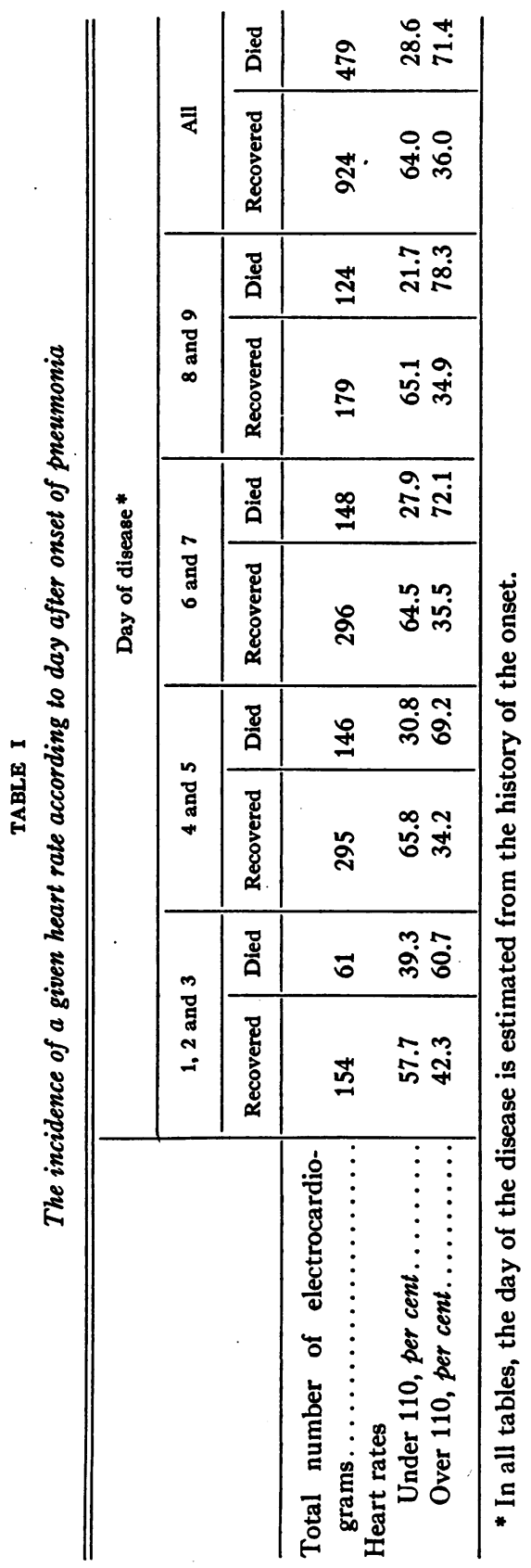


which they were taken. This was done separately for the recovered and fatal cases, and the results are shown in Table I. It may be stated generally that nearly two-thirds of the electrocardiograms taken on patients who subsequently recovered showed ventricular rates under 110 per minute, regardless of the day of the disease on which the rate was recorded; and conversely, that more than twothirds of the tracings obtained from patients who later died showed ventricular rates over 110 per minute. It may also be seen that the poor prognosis of a high ventricular rate becomes worse as it occurs later in the disease.

This conclusion suggested to us that we might arrange the data in the form of a life expectancy table (Table II). Although this is based on the number of electrocardiograms rather than on the number of patients, it clearly indicates the general trend of mortality for the different rate groups throughout the febrile period.

It is noteworthy that a fair number of tracings ( 11.3 per cent) show heart rates of 70 to 89 per minute during the period of fever. It might be assumed that in these cases the temperature was proportionately low, but as a matter of fact, in many the temperature was very high. We have selected the charts of two patients showing this. condition and have reproduced them in Figures 2 and 3.

Exceedingly slow heart rates due to sinus bradycardia were not uncommon after the temperature had become normal. At some time during convalescence, heart rates of less than sixty per minute were noted in 105 patients (32 per cent) not treated with digitalis who recovered. Nineteen patients ( 4.8 per cent) had rates between 40 . and 49 , and one showed a sinus bradycardia of 38 per minute in one tracing.

From our data we have thus far demonstrated that the heart rate is remarkably constant from day to day during the febrile period. We have also shown that with higher heart rates the expectancy of life diminishes, this relationship being even more pronounced in the later days of the disease. We have noted the occurrence of heart rates slow in proportion to fever, and of sinus bradycardia during. convalescence. 
ARThur C. DEgRAFF, JANET G. TRAVELl, AND J. ALLEN YAGER 641

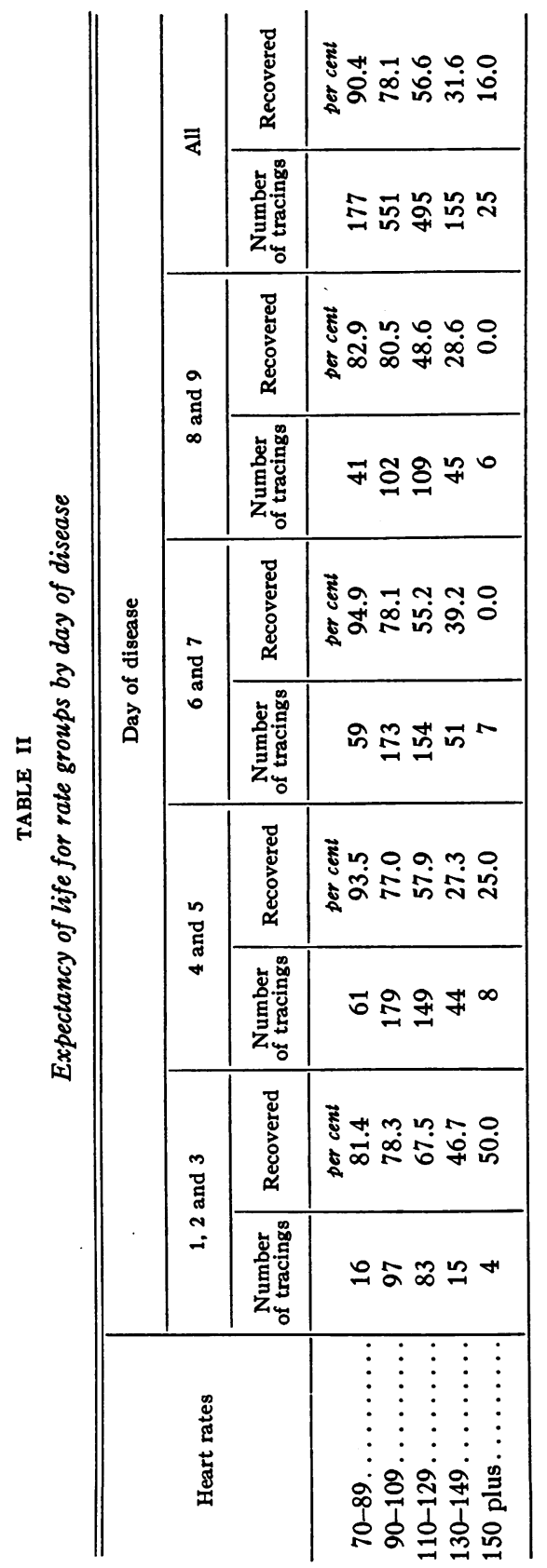




\section{Abnormalities of rhythm}

a. Abnormal basic rhythms. Auricular fibrillation and flutter in lobar pneumonia have received considerable attention, particularly from the standpoint of prognosis and treatment. In our series of 975 cases (digitalis treated and untreated) auricular fibrillation and flutter occurred in 35 instances (3.6 per cent). In additon to auricular fibrillation and flutter, we have included paroxysmal tachycardia and wandering pacemaker as disturbances of the basic rhythm of the heart. These irregularities were recorded by the electrocardiograph in 51 cases. With more frequent tracings a higher incidence would probably have been found, as all of these arhythmias were paroxysmal in nature with the exception of ten cases of persistent auricular fibrillation.

Because the number of cases in each category is so small and because the incidence is found to be approximately the same for digitalis treated and untreated groups (Wyckoff, DuBois and Woodruff (7)), in dealing with these abnormal basic rhythms we have included also the electrocardiograms of patients who received digitalis.

In the first analysis of abnormal basic rhythms (Table III), there

TABLE III

Incidence of abnormal basic rhythms in 975 cases * and associated mortality

\begin{tabular}{|c|c|c|c|c|}
\hline Type of arhythmia & Number of cases & Incidence & Number died & Mortality \\
\hline Auricular fibrillation. & 27 & $\begin{array}{c}\text { per cent } \\
2.8\end{array}$ & 17 & $\begin{array}{c}\text { per cent } \\
63.0\end{array}$ \\
\hline Auricular flutter . . . . & $10 \dagger$ & 1.0 & 7 & 70.0 \\
\hline Paroxysmal tachycardia . & 6 & 0.6 & 4 & 66.7 \\
\hline Wandering pacemaker. & 8 & 0.8 & 4 & 50.0 \\
\hline Total-all types...... & 51 & 5.2 & 32 & 62.8 \\
\hline
\end{tabular}

The mortality of the entire series excluding the patients with abnormal basic rhythms (924 cases) was 34.3 per cent.

* In this table both the digitalis treated and untreated cases are included.

$\dagger$ Two of the cases of auricular flutter also showed fibrillation and have been included in both groups.

are two outstanding facts to be noted, namely, their surprisingly low incidence and their consistently high mortality. The mortality for the various types of arhythmias ranges from 15 per cent to 35 per cent higher than the gross mortality for all cases with sinus rhythm, and 
the average mortality for all the arhythmias is 28.5 per cent higher. Since in the general series of pneumonia patients the older age groups show a much higher mortality than do the younger, a difference especially striking above and below forty, the very high figure for the arhythmias might be thought to be due to the fact that a large proportion of them occurred in the older age groups. It is indeed true that the incidence of arhythmias (Table IV) in patients over forty is

TABLE IV

The incidence and mortality of cases with abnormal basic rhythm compared with sinus rhythm for the age groups above and below forty years*

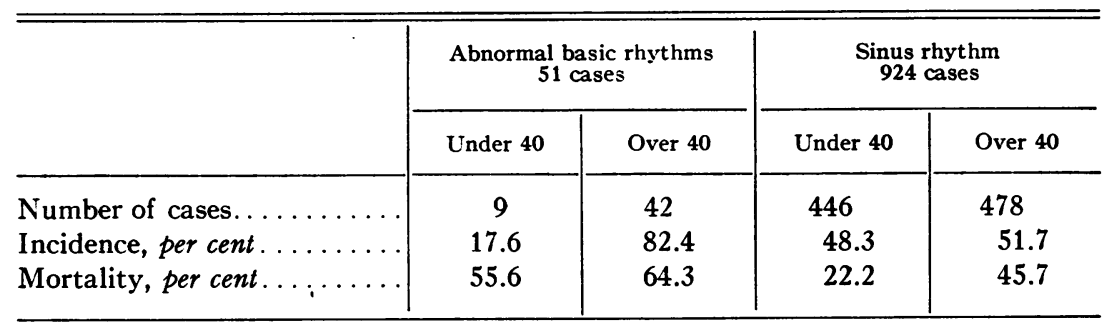

* In this table both the digitalis treated and untreated cases are included.

nearly five times that in patients below that age, while the incidence of sinus rhythm is practically the same in both age groups. However, in cases with sinus rhythm the mortality rate over forty years of age is twice that under forty, whereas the occurrence of abnormal basic rhythms raises the mortality of both age groups and makes them more nearly equal. This comparison indicates a marked increase in mortality when disturbances of basic rhythm occur regardless of the age of the patient. It was thought possible that arhythmias developed chiefly in the more severe types of pneumonic infections. Therefore, a comparative study of the incidence of the pneumococcic types was made and the distribution found to be essentially the same for these arhythmias as for sinus rhythm. One must therefore conclude that the development of an abnormal basic rhythm in the course of pneumonia seriously affects the prognosis by its own influence.

b. Interruptions of the basic rhythm. Premature contractions are to be considered as interruptions of the basic rhythm of the heart. It is obvious that for their analysis electrocardiograms taken on digitalis 
treated cases must be excluded. The different types of premature contractions have been studied separately and their incidence and mortality are shown in Table $\mathrm{V}$. The occurrence of ventricular

TABLE V

Incidence and mortality of cases with premature contractions

\begin{tabular}{c|c|c|c|c}
\hline Type of premature contraction & Number of cases & Incidence & Number died & Mortality \\
\hline Ventricular only ............ & 32 & per cent & & per cent \\
Auricular or nodal alone, or in & & 5.7 & 10 & 31.3 \\
combination with ventricular.... & 33 & 5.9 & 14 & 42.4 \\
Total-all types............. & 65 & 11.6 & 24 & 36.9 \\
\hline
\end{tabular}

premature contractions alone does not seem to influence the prognosis, for the mortality figure (31.3 per cent) of cases showing this type of premature contraction is almost identical with that of the entire series (31.2 per cent). When the auricular or nodal premature contractions occur alone, or in combination with ventricular premature contractions, the mortality rate is somewhat higher. An explanation for this is suggested by the data in Table VI, in which the association

TABLE VI

Association of abnormal basic rhythms with premature contractions

\begin{tabular}{|c|c|c|c|}
\hline \multirow{2}{*}{ Type of premature contraction } & \multirow{2}{*}{$\begin{array}{c}\text { Number } \\
\text { of } \\
\text { cases }\end{array}$} & \multicolumn{2}{|c|}{$\begin{array}{l}\text { Those associated with } \\
\text { abnormal basic rhythms }\end{array}$} \\
\hline & & Number & Per cent \\
\hline $\begin{array}{l}\text { Ventricular only } \ldots \ldots \ldots \ldots \\
\text { Auricular or nodal alone, or in combination } \\
\quad \text { with ventricular } \ldots \ldots \ldots \ldots \ldots \ldots \ldots \ldots\end{array}$ & 32 & 2 & $\begin{array}{r}6.2 \\
18.2\end{array}$ \\
\hline
\end{tabular}

of the various types of premature contractions with disturbances of the basic rhythm is tabulated. The incidence of ventricular premature contractions in association with arhythmias is low as compared with that of auricular and nodal premature contractions. This fact might account for the slightly higher mortality in the latter group. It may be of interest to note that of the 33 cases showing auricular or nodal premature contractions, 24 occurred alone and 9 in combination with the ventricular type. 
It is evident that when the basic rhythm of the heart is altered the prognosis is not nearly so good as when a sinus rhythm is continuously present. However, the occurrence of ventricular premature contractions alone should not be regarded as a poor prognostic sign.

\section{Abnormalities of conduction}

The occurrence of auriculoventricular block in patients receiving no digitalis is not rare. Using the criteria of Lewis and Gilder (9), that the upper limit of the normal $\mathrm{P}-\mathrm{R}$ interval is .20 second, in our series of 562 cases we noted prolongation of auriculo-ventricular conduction time in 51 (9.2 per cent), dropped beats in three ( 0.5 per cent), and complete dissociation in none. Cohn and Jamieson (6) did not mention the occurrence of heart block in fifty digitalis control cases, but they were primarily interested in the maximum change in the $\mathrm{P}-\mathrm{R}$ interval. They found no case in which the $\mathrm{P}-\mathrm{R}$ interval increased more than .04 seconds during fever. In two cases they found a decrease exceeding that figure. In order to make our figures comparable to a certain extent with those of Cohn and Jamieson, we have determined the maximum range in the $\mathrm{P}-\mathrm{R}$ interval for our cases of prolonged conduction time (Table VII). Over 85 per cent showed a change of .04 second or more. In Table VIII the minimum

TABLE VII

Maximum range of $P-R$ intervals in 51 cases of prolonged conduction time

\begin{tabular}{|c|c|c|c|c|c|c|c|c|c|}
\hline & \multicolumn{7}{|c|}{ Maximum range in seconds } & \multirow{2}{*}{$\underset{\text { known }}{\text { Un- }}$} & \multirow{2}{*}{ Total } \\
\hline & .03 & .04 & .05 & .06 & .07 & .08 & .09 & & \\
\hline $\begin{array}{l}\text { Number of cases. } \\
\text { Incidence, per cent }\end{array}$ & $\begin{array}{l}5 \\
9.8\end{array}$ & $\begin{array}{l}17 \\
33.3\end{array}$ & $\begin{array}{c}7 \\
13.7\end{array}$ & $\begin{array}{l}11 \\
21.6\end{array}$ & $\begin{array}{l}4 \\
7.8\end{array}$ & $\begin{array}{l}5 \\
9.8\end{array}$ & $\begin{array}{l}1 \\
2.0\end{array}$ & $\begin{array}{l}1 \\
2.0\end{array}$ & $\begin{array}{c}51 \\
100.0\end{array}$ \\
\hline
\end{tabular}

* One tracing only.

TABLE VIII

Minimum $P-R$ intervals in 51 cases of prolonged conduction time

\begin{tabular}{|c|c|c|c|c|c|c|c|c|c|c|}
\hline & \multicolumn{9}{|c|}{ Length of minimum $P-R$ interval in seconds } & \multirow{2}{*}{ Total } \\
\hline & .14 & .15 & .16 & .17 & .18 & .19 & .20 & .21 & .22 & \\
\hline Number of cases..... & 1 & 0 & 14 & 7 & 15 & 3 & 9 & 0 & $2^{*}$ & 51 \\
\hline
\end{tabular}

* One of these cases had one tracing only. 
$\mathrm{P}-\mathrm{R}$ intervals are given, and it may be seen that all but two cases showed at some time what may be considered a normal $\mathrm{P}-\mathrm{R}$ interval. From Tables IX and $\mathrm{X}$ it is shown that in $\mathbf{5 0}$ per cent of the cases

TABLE IX

Relation of increased $A-V$ conduction time to fever in 562 cases

\begin{tabular}{|c|c|c|c|}
\hline & $\begin{array}{l}\text { Febrile } \\
\text { period }\end{array}$ & $\begin{array}{c}\text { Afebrile } \\
\text { period }\end{array}$ & Totals \\
\hline 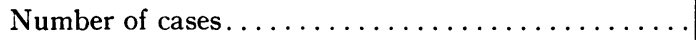 & 11 & 40 & 51 \\
\hline Percentage of cases....... & 21.6 & 78.4 & 100 \\
\hline Number died . . . . . . . . . . & 3 & 0 & 3 \\
\hline Number later showing normal $\mathrm{P}-\mathrm{R}$ interval. & 5 & 20 & 25 \\
\hline Percentage restored to normal $\mathrm{P}-\mathrm{R}$ interval. . & 45.5 & 50.0 & 49.0 \\
\hline
\end{tabular}

TABLE $\mathrm{X}$

Incidence of increased $A-V$ conduction time according to days after crisis

\begin{tabular}{|c|c|c|c|c|}
\hline \multirow{2}{*}{ Days after crisis } & \multicolumn{2}{|c|}{ Prolonged $\mathrm{P}-\mathrm{R}$ interval } & \multicolumn{2}{|c|}{ Restored to normal $\mathrm{P}-\mathrm{R}$ interval } \\
\hline & $\begin{array}{l}\text { Number of } \\
\text { cases }\end{array}$ & $\begin{array}{c}\text { Incidence in } \\
40 \text { cases }\end{array}$ & $\begin{array}{l}\text { Number of } \\
\text { cases }\end{array}$ & Percentage \\
\hline $\begin{array}{l}1,2,3 \ldots \ldots \\
4,5,6 \ldots \ldots \\
7,8,9 \ldots \ldots \\
10,11,12 \ldots \ldots \\
13,14 \ldots \ldots \\
\text { All days. } \ldots \ldots\end{array}$ & $\begin{array}{r}22 \\
7 \\
6 \\
1 \\
4 \\
40\end{array}$ & $\begin{array}{r}\text { per cent } \\
55.0 \\
17.5 \\
15.0 \\
2.5 \\
10.0 \\
100.0\end{array}$ & $\begin{array}{r}11 \\
1 \\
5 \\
1 \\
2 \\
20\end{array}$ & $\begin{array}{c}\text { per cent } \\
50.0 \\
14.3 \\
83.3 \\
100.0 \\
50.0 \\
50.0\end{array}$ \\
\hline
\end{tabular}

Note: There were no cases in which the $\mathrm{P}-\mathrm{R}$ interval first became prolonged later than the 14th day after the crisis.

with increased $\mathrm{A}-\mathrm{V}$ conduction time, the $\mathrm{P}-\mathrm{R}$ interval was restored to normal at some time during our observation. This seems to indicate that the changes in conduction are definitely related to the disease.

In certain other diseases, namely; rheumatic fever and diphtheria, electrocardiographic changes found in the afebrile period are admitted as evidence that the myocardium has in some way been involved. Cohn and Jamieson (6) excluded from the analysis of their pneumonia series all cases in which the increase in conduction time occurred 
during the postcritical period, whereas we have included them. Cohn and Swift (1) in a report on the electrocardiographic evidence of myocardial involvement in rheumatic fever consider a prolongation of the $\mathrm{P}-\mathrm{R}$ interval during the afebrile period, as well as during the febrile period, as evidence of myocardial involvement. Heart block in diphtheria occurs not only in the acute stages but later in the disease; for instance, Parkinson (10) reports a case of heart block occurring on the twenty-third day. It therefore seems reasonable to consider as significant, similar changes occurring in lobar pneumonia. In Table IX we have compared the incidence of prolonged conduction time before and after defervescence. Although the major part (78 per cent) first shows a prolongation of the $\mathrm{P}-\mathrm{R}$ interval after the temperature has reached a normal level, still in an appreciable number (22 per cent) block first appears during fever. In the latter group of eleven, nine cases had a maximum conduction time of .22 second and two, of .24 second.

In the postfebrile period auriculoventricular block occurs first very soon after the temperature has become normal. In Table $\mathrm{X}$ we have correlated the first appearance of increased conduction time in the convalescent period with the number of days elapsed after the crisis or, if defervescence was by lysis, with the number of days after the temperature became normal. Over half of the patients developed an increased $\mathrm{P}-\mathrm{R}$ interval during the first three days of convalescence and in approximately one-half of these cases the conduction times returned to normal while the patients were still under our observation.

The factor of heart rate must be considered in relation to prolonged auriculoventricular conduction time. Lewis and Cotton (11) have shown that after exercise, the increase in heart rate is accompanied by a reduction in the $\mathrm{P}-\mathrm{R}$ interval. Cohn and Jamieson (6) noted that at the height of the fever in those cases of lobar pneumonia showing a rapid heart rate, there was a reduction in the auriculoventricular conduction time. We have correlated heart rate with increased conduction time, and in 34.7 per cent of our cases showing this change, increasing $\mathrm{P}-\mathrm{R}$ interval occurred simultaneously with slowing of the heart rate. However, in 52.1 per cent no such correlation could be found and in 13.2 per cent the P-R interval actually increased as the ventricular rate increased. 
Various grades of intraventricular block, and in a few cases complete bundle branch block, were found in both digitalis treated and untreated groups. In the first two years of this study measurements were not made of the duration of the Q.R.S. group and only frank cases of complete bundle branch block were noted. There were two such cases, one in each year. Both had the same degree of bundle branch block throughout and both died. It is possible that the block existed before the pneumonia developed and that the occurrence here is merely a coincidence. In the third year, accurate measurements of the width of the Q.R.S. group were made on every tracing. A total of nineteen cases ( 9.3 per cent) were found with Q.R.S. width exceeding .10 second during some part of the disease. The mortality in this group was only 26.8 per cent, which compares favorably with the gross mortality for the third year of 28.2 per cent. Eleven of these cases had a prolonged Q.R.S. group on the first tracing and continued to show intraventricular block on each subsequent tracing. It is likely that the intraventricular block antedated the pneumonia. Incidentally, only three of the eleven patients died, a surprisingly low mortality, considering the significance that is ordinarily attached to a wide Q.R.S. group. The other eight patients developed intraventricular block during the course of the disease. Two of these patients died. It is noteworthy that two patients did not develop an intraventricular block until after the temperature had become normal, indicating again that active changes in the heart do not terminate with the crisis.

\section{Changes in the $S-T$ segment and $T$ wave}

An individual case in which there was marked elevation of the $\mathrm{R}-\mathrm{T}$ segment with plateau-like $\mathrm{T}$ wave has already been reported by Shearer (12). A few cases showing similar changes of much less marked degree were also noted.

A change in direction of the $T$ wave from an upright to an inverted position in Lead III of the electrocardiogram was observed in 14.4 per cent of patients not treated with digitalis, and in Lead I or in a combination of Leads I and II, or II and III, in 2.4 per cent of cases. T wave inversion in Lead III alone is known to be associated frequently with respiratory changes, but inversions in other leads are more probably related directly to the pneumonia. 
Other changes in the form of the $T$ wave and $S-T$ segment were observed, but because of the lack of sufficient data with reference to the degree of variability of their form in the normal electrocardiogram, a detailed analysis has been avoided.

\section{Serum therapy and the electrocardiogram}

About one-third of the cases studied in our series received intensive treatment with specific antipneumococcus serum. Thus, the possible influence of the administration of serum on the electrocardiogram was investigated. The incidence of abnormalities of rate, rhythm and conduction was found to be practically the same in the serum treated and untreated groups. Increased auriculoventricular conduction time during fever occurred somewhat more frequently in serum treated cases. However, there being a total of only eleven such cases, this fact cannot be made significant. It may therefore be stated generally that the serum administered does not influence the electrocardiographic findings.

\section{SUMMARY}

From the data we have presented it is evident that although electrocardiographic changes in lobar pneumonia are not so frequent as in certain other diseases, notably rheumatic fever and diphtheria, they occur often enough to be of significance. In approximately onequarter of all our cases, transitory changes were detected by the electrocardiogram at some time in the course of the disease: 10 per cent showed abnormalities of rhythm; 10 per cent showed disturbances of conduction; and in a small proportion, 2.4 per cent, inversions of the $T$ wave other than in Lead III were noted.

A remarkable fact is that many of these changes occurred first in the postfebrile period. Marked sinus bradycardia, prolongation of auriculoventricular conduction time and intraventricular block occurring during convalescence have already been discussed. Also in two instances auricular flutter appeared after the temperature had begun to fall by lysis, and in the case reported by Shearer, the maximum degree of change in the $\mathrm{R}-\mathrm{T}$ segment did not develop until the temperature had begun to fall. The evidence seems to indicate that whatever changes the pneumonic infection produces in the heart, these changes persist into, and may appear for the first time, in the afebrile period. 
While specific pathological changes in the heart have not yet been demonstrated in pneumonia, we do not feel that this militates against the interpretation of our findings. We do not attempt to place these observations on any definite structural basis.

\section{CONCLUSIONS}

1. The heart rate is a valuable guide to prognosis throughout the course of lobar pneumonia. The expectancy of life diminishes as the heart rate increases, this correlation being even more pronounced in the later days of the disease.

2. The heart rate in pneumonia in a large proportion of cases remains fairly constant during the febrile period, with only slight variations from day to day and in many instances despite fluctuations of temperature.

3. Slow heart rates may occur in pneumonia even in the presence of high temperatures.

4. Abnormalities of rhythm occur in about 10 per cent of our cases.

5. Abnormal basic rhythms are found in about 5 per cent of cases, and in these the prognosis is poor.

6. The occurrence of premature contractions of ventricular origin does not alter the prognosis. Premature contractions of auricular or nodal origin may lessen the chances of recovery.

7. Conduction defects in lobar pneumonia occur in about 10 per cent of our patients not treated with digitalis.

8. In lobar pneumonia disease processes are active in the heart even after the temperature has become normal. Evidence to support this statement is found in the occurrence of auriculoventricular and intraventricular block and sinus bradycardia in the afebrile period of the disease.

\section{BIBLIOGRAPHY}

1. Cohn, Alfred E. and Swift, Homer F., J. Exp. Med., 1924, xxxix, 1. Electrocardiographic Evidence of Myocardial Involvement in Rheumatic Fever.

2. Rothschild, M. A., Sacks, B. and Libman, E., Am. Heart J., 1927, ii, 356. The Disturbances of the Cardiac Mechanism in Subacute Bacterial Endocarditis and Rheumatic Fever.

3. Marvin, Harold M., Am. J. Dis. Child., 1925, xxix, 433. The Effect of Diphtheria on the Cardiovascular System. I. The Heart in Faucial Diphtheria. 
4. McCulloch, H., Am. J. Dis. Child., 1920, xx, 89. Studies on the Effect of Diphtheria on the Heart.

5. Smith, S. C., J. Am. Med. Assoc., 1921, lxxvii, 765. Observations on the Heart in Diphtheria.

6. Cohn, Alfred E. and Jamieson, Ross A., J. Exp. Med., 1917, xxv, 65. The Action of Digitalis in Pneumonia.

7. Wyckoff, John, DuBois, Eugene F. and Woodruff, I. Ogden, J. Am. Med. Assoc., 1930, xcv, 1243. The Therapeutic Value of Digitalis in Pneumonia.

8. Bullowa, Jesse G. M., J. Am. Med. Assoc., 1928, xc, 1354 . Use of Antipneumococcic Refined Serum in Lobar Pneumonia.

9. Lewis, Thomas and Gilder, M. D. D., Phil. Trans. Roy. Soc. Series B, 1912, ccii, 351. The Human Electrocárdiogram: A Preliminary Investigation of Young Male Adults to Form a Basis for Pathological Study.

10. Parkinson, John, Heart, 1915, vi, 13. Auricular Fibrillation Following Complete Heart Block in Diphtheria.

11. Lewis, Thomas and Cotton, T. F., (Proc. Physiol. Soc., June 28, 1913), J. Physiol., 1913, xlvi, lx. The P-R Interval in Human Electrocardiograms, and Its Relation to Exercise.

12. Shearer, Margery C., Am. Heart J., 1930, v, 801. Plateau R-T in a Case of Lobar Pneumonia. 